\title{
Adaptação e adaptação cruzada de Listeria monocytogenes aos compostos eugenol e carvacrol
}

SOUZA, E.R.N.'; TEBALDI, V.M.R.2; PICCOLI, R.H. ${ }^{*}$

${ }^{1}$ Departamento de Ciência dos Alimentos - Universidade Federal de Lavras, Caixa Postal 3037, 37.200-000, MG, Brasil, souzae78@yahoo.com.br, 2Departamento de Biologia, Microbiologia Agrícola - Universidade Federal de Lavras, Caixa Postal 3037, 37.200-000,MG, Brasil, victormaxibio@yahoo.com.br, ${ }^{3}$ Departamento de Ciência dos Alimentos - Universidade Federal de Lavras, Caixa Postal 3037, 37200-000,MG, Brasil, *Autor para correspondência: rhpiccoli@dca.ufla.br

RESUMO: A evolução de certos microrganismos permite sua rápida adaptação aos ambientes em constante mudança, desenvolvendo assim, tolerância ou resistência ao aumento de determinados estresses. $\mathrm{O}$ uso de compostos bioativos provenientes da flora nativa tem sido apontado como uma possível solução para os problemas de controle da resistência e proliferação bacteriana. Este trabalho visou verificar a adaptação e adaptação cruzada de L. monocytogenes, frente aos compostos fenólicos eugenol e carvacrol. A concentração mínima inibitória (CMI) dos compostos fenólicos foi determinada pela técnica de microdiluição em placas de 96 cavidades, em caldo TSB + 0,5\% de Tween 80 . As concentrações finais (\%) obtidas foram: 0,06; 0,12; 0,24; 0,$49 ; 0,98 ; 1,95 ; 3,9 ; 6,25 ; 12,5 ; 25 ; 50$. A suspensão bacteriana padronizada foi inoculada nas cavidades das placas, as quais foram incubadas a $37^{\circ} \mathrm{C}$ por 24 horas com posterior leitura da absorbância a $620 \mathrm{~nm}$ e determinação da CMI. A adaptação das células de L. monocytogenes ao eugenol e carvacrol foi realizada com o cultivo das células em TSB $+0,06 \%$ de eugenol ou carvacrol à $37^{\circ} \mathrm{C}$ por 2 horas. A cultura foi então centrifugada e as células ressuspendidas e padronizadas em TSB. A seguir, realizou-se novamente a técnica de microdiluição em caldo. Os resultados obtidos demonstraram que $L$. monocytogenes apresentou adaptação e adaptação cruzada frente ao carvacrol e eugenol. A CMI do eugenol e carvacrol foi de $24 \%$. A pré-exposição de $L$. monocutogenes a concentração sub-letal de $0,06 \%$ de carvacrol ou de eugenol aumentou sua resistência. A pré-exposição ao carvacrol promoveu a adaptação de L.monocytogenes a ele aumentando a CMI para $12,5 \%$. Já para o eugenol a CMI passou para $25 \%$. Quando submetidas à concentração sub-letal de eugenol, este promoveu a adaptação das células tanto ao carvacrol quanto ao eugenol, sendo a $\mathrm{CMI}$ de $12,5 \%$. Os resultados obtidos demonstraram que $L$. monocytogenes apresentou adaptação e adaptação cruzada ao carvacrol e eugenol. $\mathrm{O}$ presente trabalho sugere estudos futuros ainda mais abrangentes quanto à potencialidade antimicrobiana destes compostos.

Palavras-chave: antimicrobianos naturais, compostos bioativos, patógeno alimentar

\begin{abstract}
Adaptation and Cross adaptation of Listeria monocytogenes to eugenol and carvacrol compounds. Some microorganisms have evolved and therefore are able to rapidly adapt themselves to a constantly changing environment, thus developing tolerance or resistance to the increase of some specific stresses. The use of bioactive compounds from the native vegetation has been pointed out as a possible solution to the problems of control of bacterial resistance and proliferation. This work aimed to check the adaptation and cross adaptation of L. monocytogenes, towards the phenolic compounds eugenol and carvacrol. The minimum inhibitory concentration (MIC) of the phenolic compounds was determined by the microdilution technic in plates of 96 cavities, in CALDO TSB $+0,5 \%$ of Tween 80 . The final concentrations (\%) obtained were: 0,$06 ; 0,12 ; 0,24 ; 0,49 ; 0,98 ; 1,95 ; 3,9 ; 6,25 ; 12,5 ; 25 ; 50$. The patronized bacterial suspension was inoculated into the captivities of the plates, and incubated at $37^{\circ} \mathrm{C}$ for 24 hours with posterior reading of the absorbance at $620 \mathrm{~nm}$ and determination of the MIC. The adaptation of the cells of $L$. monocytogenes to the eugenol and carvacrol was made through the cultivation of the cells in TSB $+0,06 \%$ of eugenol or carvacrol at $37^{\circ} \mathrm{C}$ for 2 hours. The sample
\end{abstract}


was then centrifuged and the cells were re-suspended and patronized in TSB. After that, the microdilution technic was performed one more time. The obtained results revealed that the L. monocytogenes presented adaptation and cross adaptation to the eugenol and carvacrol. The eugenol and carvacrol CMI was at $24 \%$. The pre-exposition of $L$. monocytogenes to sublethal doses of $0,06 \%$ of eugenol or carvacrol enhanced its resistance. The pre-exposition to carvacrol promoted the adaptation of $L$. monocytogenes to it thus increasingthe MIC to $12,5 \%$. To the eugenol the CMl got to $25 \%$. When submitted to sub-lethal concentrations of eugenol, the latterpromoted the adaptation of the cells to carvacrol and eugenol, bringing the CMI to $12,5 \%$. The obtained results showed that the L. monocytogenespresented adaptation and cross adaptation to the eugenol and carvacrol. The current work suggests future studies even broader regarding the antimicrobial potentialityof these compounds.

Keywords: natural antimicrobial, bioactive compounds, food pathogen.

\section{INTRODUÇÃO}

Apesar das melhorias nas condições higiênico-sanitárias no processamento de alimentos em países industrializados, surtos de listeriose originados pelo consumo de alimentos contaminados ainda ocorrem e asseguram que o controle efetivo de Listeria monocytogenes mostra-se ser um desafio (Harvey et al., 2007).

A Organização Mundial da Saúde considera L. monocytogenes um dos cinco mais importantes microrganismos causadores de doenças transmitidas por alimentos. A listeriose é uma doença de origem alimentar atípica, devido à alta gravidade, natureza não entérica e longo período de incubação (Loguercio et al., 2001).

Embora haja mais de 20 anos de discussões a respeito dos critérios microbiológicos a respeito de L. monocytogenes em alimentos a serem estabelecidos, e a Comissão do Codex Alimentarius já ter se pronunciado, as discussões ainda continuam (Andersen \& Norrung, 2011).

Listeria monocytogenes possui elevada resistência fisiológica, sendo difícil controlar ou prevenir sua presença em alimentos, principalmente naqueles que não sofrem tratamento térmico (Lukinmaa et al., 2004, Samelis et al., 2005). Várias cepas são resistentes aos antibióticos usuais, tornando a sua ocorrência em alimentos e superfícies de processamento ameaça potencial à saúde (Ribeiro et al., 2006; Mantilla et al., 2008).

A resistência de cepas de $L$. monocytogenes a antibióticos pode comprometer as opções de tratamentos. Segundo Conter et al. (2009), existe pouca informação sobre a susceptibilidade a antimicrobianos de cepas de L. monocytogenes isoladas de alimentos e ambientes de produção.

O controle de microrganismos é um dos mais importantes aspectos na conservação de alimentos e saúde pública. Temperatura, atividade de água, adição de conservantes e pH são alguns dos parâmetros utilizados para inibir ou destruir microrganismos e como auxílio na conservação de alimentos (Beales, 2004). Entretanto, a maioria dos antimicrobianos, conservantes e sanificantes, usados na produção de alimentos têm sido usados por mais de 50-100 anos, sendo, atualmente, consenso o número crescente de microrganismos patogênicos que vem desenvolvendo de resistência a esses compostos (Davidson \& Harrison, 2002).

A adaptação e resistência de $L$. monocytogenes a desinfetantes influenciam a sobrevivência da bactéria nas plantas de processamento de alimentos (Aese et al., 2000; Mereghetti et al., 2000). Os sanificantes são amplamente utilizados em instalações de processamento de alimentos, contudo, apesar da realização do processo de higienização regular, a eliminação de $L$. monocytogenes tem sido cada vez mais difícil (Lundén et al., 2003).

Estudos têm mostrado que a maior persistência da bactéria em ambiente de processamento está associada a sua constante exposição a condições subletais de fatores estressantes (antimicrobianos, $\mathrm{pH}$, temperatura, etc) fato que induz seu desenvolvimento e crescimento condicionado ao estresse, tornando-a fisiologicamente mais tolerante a níveis aumentados do mesmo ou de diferentes fatores estressantes, como mostrado por Gahan et al (1996), onde culturas de $L$. monocytogenes, isoladas de ambientes de processamento, após serem expostas a estresse subletal ácido apresentaram resistência aumentada (adaptação) a níveis maiores de acidez, e se tornaram também mais tolerantes aos estresses térmico e osmótico (adaptação cruzada). Lou \& Yousef (1997) também observaram a adaptação e adaptação cruzada de L. monocytogenes a agentes estressantes encontrados em ambientes de processamentos de alimentos e alimentos como etanol, peróxido de hidrogênio e cloreto de sódio.

A adaptação e adaptação cruzada também

Rev. Bras. PI. Med., Campinas, v.17, n.4, p.528-533, 2015. 
foram observadas para $L$. monocytogenes após sua constante exposição a concentrações subletais de sanificantes, aumentando sua sobrevivência nas condições estressantes dos ambientes de processamento e alimentos (Carpentier \& Cerf, 2011).

Assim, o conhecimento da possível adaptação cruzada de L. monocytogenes a desinfetantes seria valioso ao projetar rotação de desinfetantes no processamento de alimentos (Lundén et al., 2003).

Diante do aumento de casos de infecções de natureza bacteriana resistente e da dificuldade do controle da proliferação bacteriana em instalações da indústria alimentícia, o uso de bioativos provenientes da flora nativa, tem sido apontado como possível solução para esse problema (Dantas et al., 2010).

Os compostos fenólicos das especiarias que contém alta porcentagem de eugenol e carvacrol são os principais responsáveis pela ação bactericida/bacteriostática dos óleos essenciais (Dorman et al., 2000).

No entanto, apesar de sua composição e atividades biológicas apontarem para aplicações práticas no controle de patógenos humanos na indústria de alimentos e hospitais, a ação de óleos essenciais ou seus compostos sobre bactérias clinicamente importantes ainda não foi devidamente avaliada.

Nesse contexto, esse trabalho teve como objetivo verificar a adaptação e adaptação cruzada de Listeria monocytogenes, aos compostos fenólicos eugenol e carvacrol.

\section{MATERIAL E MÉTODOS \\ Microrganismo, manutenção e padronização do inoculo}

A bactéria utilizada foi Listeria monocytogenes ATCC 19117. A cultura estoque foi mantida em meio de congelamento (glicerol - $15 \mathrm{~mL}$; peptona bacteriológica $-0,5 \mathrm{~g}$; extrato de levedura $-0,3 \mathrm{~g} ; \mathrm{Na} \mathrm{Cl}-0,5 \mathrm{~g}$; água destilada $-100 \mathrm{~mL}$ ) a $-18^{\circ} \mathrm{C}$.

Para reativação da cepa, foram inoculados $10 \mu \mathrm{L}$ da cultura estoque em tubos contendo $3 \mathrm{~mL}$ de caldo TSB (Triptone Soy Broth) e incubados por 24 horas a $37^{\circ} \mathrm{C}$. Após a incubação, foram retirados $10 \mu \mathrm{L}$ de inoculo e transferido para $200 \mathrm{~mL}$ de TSB. A cultura bacteriana foi padronizada em 108 UFC/ $\mathrm{mL}$ pelo acompanhamento do crescimento em espectrofotômetro $\left(\mathrm{DO}_{600 \mathrm{~nm}}\right)$ e contagem de UFC em TSA (Agar Triptona de Soja).

\section{Determinação da Concentração Mínima}

\section{Inibitória (CMI)}

A concentração mínima inibitória dos compostos fenólicos carvacrol e eugenol, foi determinada empregando-se a técnica de microdiluição em caldo TSB, em placas de 96 cavidades, de acordo com o NCCLS (M7-A6) (NCCLS, 2003).

Para tanto, $100 \mu \mathrm{L}$ de TSB acrescido de $0,5 \%$ de Tween 80 foram adicionados em cada cavidade da placa de microdiluição. Em seguida 100 $\mu \mathrm{L}$ das soluções de eugenol (50\%) ou carvacrol (50\%) foram acrescidos às cavidades correspondentes a coluna 1 e linha A, transferindo-se $100 \mu \mathrm{L}$ para linha seguinte após a homogeneização e assim sucessivamente, desprezando-se os $100 \mu \mathrm{L}$ finais, obtendo-se assim, as concentrações finais (\%) de composto majoritário $(0,06 ; 0,12 ; 0,24 ; 0,49 ; 0,98$ $; 1,95 ; 3,9 ; 6,25 ; 12,5 ; 25 ; 50)$. Alíquotas de $10 \mu \mathrm{L}$ de suspensão bacteriana previamente padronizada foram inoculadas nas cavidades.

Foram realizadas duplicatas e utilizados três controles para cada composto testado: sendo dois controles negativos (um contendo TSB acrescido de $0,5 \%$ de Tween 80 e composto fenólico; um contendo TSB acrescido de $0,5 \%$ de Tween 80 , inoculo e clorexidina a $0,012 \%$ ); e um controle positivo (contendo somente TSB acrescido de 0,5\% de Tween 80 e inoculo).

As placas de microdiluição foram vedadas e incubadas a $37^{\circ} \mathrm{C}$ por 24 horas. Após esse período, foi realizada a leitura da absorbância a 620 nm e determinação da concentração mínima inibitória (menor concentração de composto capaz de inibir completamente o crescimento de $L$. monocytogenes).

\section{Adaptação e Adaptação Cruzada}

A adaptação das células de $L$. monocytogenes aos compostos eugenol e carvacrol foi realizada a partir de uma cultura padronizada com 24 horas de incubação a $37^{\circ} \mathrm{C}$ em TSB. Foi transferido $1 \mathrm{~mL}$ para $200 \mathrm{~mL}$ de meio TSB, acrescido de $0,06 \%$ de eugenol ou carvacrol e reincubado à $37^{\circ} \mathrm{C}$ por 2 horas. Em seguida, a cultura foi centrifugada a $5000 \mathrm{X}$ g por 10 minutos (SIGMA 6- 15), o sobrenadante desprezado e a cultura padronizada com ressuspensão em TSB.

Nesta etapa do estudo utilizou-se a metodologia da microdiluição em caldo TSB, em placas de 96 cavidades.

Após, realizou-se a diluição de $100 \mu \mathrm{L}$ de eugenol ou carvacrol $50 \%$ em $100 \mu \mathrm{L}$ de TSB, resultando nas concentrações finais (\%) 0,06 ; 0,$12 ; 0,24 ; 0,49 ; 0,98 ; 1,95 ; 3,9 ; 6,25 ; 12,5 ; 25$; 50. Em seguida, $10 \mu \mathrm{L}$ da suspensão bacteriana padronizada, cultivada em presença de $0,06 \%$ de eugenol ou carvacrol por 2 horas, foram inoculadas

Rev. Bras. PI. Med., Campinas, v.17, n.4, p.528-533, 2015. 
nas cavidades das microplacas, e incubadas a $37^{\circ} \mathrm{C}$ por 24 horas. Após, realizou-se a leitura da absorbância a $620 \mathrm{~nm}$.

\section{RESULTADOS E DISCUSSÃO}

A existência de cepas de $L$. monocytogenes resistentes aos antimicrobianos utilizados rotineiramente no tratamento da listeriose representa problema para a saúde pública.

A literatura é abundante em relatos da resistência de Listeria sp. à antibióticos. Porém, alguns poucos trabalhos relatam sobre a resistência desses microrganismos a óleos essenciais e seus compostos e informações sobre os efeitos destes compostos naturais sobre microrganismos de origem alimentar são limitadas.

Os valores de CMI (Concentração Mínima Inibitória) dos compostos eugenol e carvacrol obtidos nesse experimento, estão expressos na Tabela 1.

Os valores do ensaio de adaptação e adaptação cruzada de Listeria monocytogenes aos compostos carvacrol e eugenol, obtidos no experimento estão expressos na Tabela 2.

A pré incubação de L. monocytogenes em presença de $0,06 \%$ de carvacrol ou $0,06 \%$ de eugenol, aumentou sua resistência as condições letais dos antimicrobianos. Observa-se que a pré-exposição da L. monocytogenes ao carvacrol promoveu uma adaptação a ele aumentando a CMI de $0,24 \%$ para $12,5 \%$. Já a CMI do eugenol passou de $0,24 \%$ para $25 \%$.

Os compostos fenólicos das especiarias que contêm alta percentagem de eugenol, carvacrol e/ou timol, são os principais responsáveis pela sua ação bactericida/bacteriostática. Porém, as concentrações antimicrobianas de cada composto variam. Os resultados obtidos nesse estudo demonstraram que $L$. monocytogenes apresentouse sensível ao eugenol e carvacrol a partir da concentração $0,24 \%$.

Os óleos essenciais são lipofílicos, penetrando no envelope celular e membrana citoplasmática, destruindo a camada de polissacarídeos, ácidos graxos e fosfolípideos, alterando sua permeabilidade. Essa alteração leva à perda de íons com perda do potencial de membrana, colapso da bomba de prótons e depleção de ATP, coagulação citoplasmática e clivagem de macromoléculas (Solomakos et al., 2008). Estudos mostram que os compostos fenólicos dos óleos essenciais se ligam à bicamada fosfolipídica da membrana celular aumentando sua permeabilidade e promovendo o extravasando dos constituintes intracelulares ou danificando o sistema enzimático da célula (Moreira et al., 2005). Especificamente o carvacrol e o timol podem interferir no transporte de elétrons, absorção de nutrientes, síntese de ácidos nucleicos e também interagir com proteínas da membrana, causando deformação e prejudicando sua funcionalidade (Bajpai et al., 2008).

Devido ao seus múltiplos modo de ação, os óleos essenciais e seus compostos têm sido sujeridos como antimicrobianos alternativos em detrimento aos comumente utilizados, cujo alvo na célula normalmente é único. Entretanto trabalhos devem ser realizados para garantir a segurança de sua utitlização na indústria de alimentos.

Devido a exposição a múltiplos fatores de estresse ambientais, que mudam sucessivamente durante o processamento, as células bacterias

TABELA 1. Concentração Mínima Inibitória de eugenol e carvacrol sobre Listeria monocytogenes

\begin{tabular}{|c|c|c|}
\hline \multirow{2}{*}{ Composto } & \multicolumn{2}{|c|}{ Concentração (\%) } \\
\hline & \multicolumn{2}{|c|}{502512,5 6,25 3,9 1,950,98 0,49 0,24 0,12 0,06 } \\
\hline Eugenol & \multicolumn{2}{|c|}{$---------^{++}$} \\
\hline Carvacrol & \multicolumn{2}{|c|}{---------++} \\
\hline \multicolumn{3}{|c|}{ (-) não crescimento; (+) crescimento } \\
\hline \multicolumn{3}{|c|}{$\begin{array}{l}\text { TABELA 2. Adaptação e adaptação cruzada de Listeria monocytogenes ao carvacrol e eugenol após exposição } \\
\text { a concentrações subletais de } 0,06 \% \text { de carvacrol e eugenol }\end{array}$} \\
\hline \multirow{2}{*}{ Estresse subletal } & \multicolumn{2}{|c|}{ Concentração mínima inibitória (\%) } \\
\hline & Carvacrol & Eugenol \\
\hline Carvacrol & 12,5 & 25 \\
\hline Eugenol & 12,5 & 12,5 \\
\hline
\end{tabular}

Rev. Bras. PI. Med., Campinas, v.17, n.4, p.528-533, 2015. 
devem se adaptar a essas condições adversas por questão de sobrevivência.

Nesse trabalho, L.monocytogenes apresentou a capacidade de adaptação e adaptação cruzada aos compostos carvacrol e eugenol. Entretanto, Apolonio et al. (2014) mostraram que o isolados de L. monocytogenes e Staphyloccoccus aures que utilizaram em seu trabalho não foram capazes de se adaptar ao eugenol e ao citral.

A adaptação de L. monocytogenes a exposição ao estresse é alcançada por mudanças na expressão global de grande número de componentes celulares. Da mesma forma, a adaptação cruzada da bactéria exposta a diferentes estresses ambientais pode ser conferida pela ação de vários mecanimos metabólicos que parecem ser ativados pelos diferentes estresses (Soni et al., 2011). Geralmente quando as bactérias são expostas ao estresse induzido por substâncias tóxicas ocorre o aumento na síntese das proteínas de estresse ou HSPs. Essas proteínas (HSPs) pertencem as famílias das chaperones tendo papel importante na montagem de polipeptídeos recém sintetizados, em sua conformação nativa e no dobramento e reparação das proteínas do citosol (Di Pasqua et al., 2010)

A adaptação de L.monocytogenes ao carvacrol e eugenol e a adaptação cruzada pode ter ocorrido por diversos fatores. Um deles poderia ser como o ocorrido em Bacillus cereus, onde seu cultivo em presença de concentrações subletais de carvacrol promoveu a diminuição da fluidez da membrana citiplasmática devido a alterações na constituição dos acidos graxos. Além disso, observou-se que o composto, embora tenha alterado a composição da membrana não foi metabolizado (Utlee et al., 2000)

Outro mecanismo seria as diversas modificações que os compostos podem causar no metabolismo celular e síntese de proteínas associadas ao estresse como mostrado ocorrer com Salmonella. Thompson após seu cultivo em concentração subletal de timol.

Salmonella Thompson após ser tratada com concentração subletal de timol apresentou grande variação na expressão de proteínas alterando também diferentes vias do metabolismo celular. Muitas proteínas foram superproduzidas outras tiveram sua produção reduzida drasticamente com mudanças significativas das suas classes funcionais. Diferentes proteínas chaperones foram super produzidas bem como aquelas associadas ao mecanismo de proteção ao estresse térmico (Di Pasqua et al., 2010).

Embora muito se tenha publicado sobre adaptação e adaptação cruzada das bactérias às condições de estresse e estresse subletal, pouco se tem a respeito dos óleos essenciais, que conforme discutido podem ou não promover o processo de adaptação e adaptação cruzada. Assim, trabalho envolvendo estudos proteômicos devem ser conduzidos para maiores conhecimentos.

\section{CONCLUSÃO}

Os resultados obtidos demonstraram que Listeria monocytogenes apresentou adaptação e adaptação cruzada frente ao carvacrol e eugenol.

\section{AGRADECIMENTOS}

Os autores agradecem à FAPEMIG pelo auxílio financeiro e a CAPES pela bolsa de estudos.

\section{REFERÊNCIAS}

AESE, B., et al. Occurrence of and possible mechanism for resistance to a quaternary ammonium compound in Listeria monocytogenes. International Journal of Food Microbiology. v. 62, 57-63, 2000.

ANDERSEN, J.K.; NORRUNG, B.. The challenge of setting risk-based microbiological criteria for Listeria monocytogenes. Food Control . v. 122, p. 1495-1497, 2011

APOLONIO, J.; et al. No induction of antimicrobial resistance in Staphylococcus aureus and Listeria monocytogenes during continuous exposure to eugenol and citral. FEMS Microbiology Letters. v. 354, p. 92-101, 2014.

BAJPAI, V.K., et al. Chemical composition and inhibitory parameters of essential oil and extracts of Nandina domestica Thunb. to control foodborne pathogenic and spoilage bacteria. International Journal of Food Microbiology, v.125, n.2, p.117-22, 2008.

BEALES, N. Adaptation of Microorganisms to Cold Temperatures, Weak Acid Preservatives, Low pH, and Osmotic Stress: A Review. Comprehensive Reviews in Food Science and Food Safety, v.3, n. 1, 1-20, 2004.

CARPENTIER, B.; CERF, O. Review - Persistence of Listeria monocytogenes in food industry equipment and premises. International Journal of Food Microbiology. v. 145, p. 1-8, 2011.

CONTER, M., et al. Characterization of antimicrobial resistance of foodborne Listeria monocytogenes. International Journal of Food Microbiology, v. 128, n. 3, 497-500, 2009.

DANTAS, L.I.S., et al. Atividade antibacteriana do óleo essencial de Lippia gracilis Schauer sobre patógenos de importância na indústria de alimentos. HOLOS, v. 5, n. 26, 2010.

DAVIDSON, P.M.; HARRISON, A.M. Resistence and adaptation to food antimicrobials, sanitizers and others process controls. Food Technology. V. 56,n.11, p. 69-78,2002.

DI PASQUA, R., et al. Changes in the proteome of Salmonella enterica serovar Thompson as stress adaptation to sublethal concentrations of thymol. Proteomics. v. 10, n. 5, p. 1040-1049, 2010.

DORMAN, H.J.D. et al. Antimicrobial agents from plants: 
antibacterial activity of plant volatile oil. Journal of Applied Microbiology, v.83, p.308-316, 2000.

GAHAN, C. G., et al. Acid adaptation of Listeria monocytogenes can enhance survival in acidic foods and during milk fermentation. Applied Environmental Microbiology. v. 62, p. 3128-3132, 1996.

HARVEY, J., et al. Assessing biofilm formation by Listeria monocytogenes strains. Food Microbiology, v. 24, n. 4, 380-392, 2007.

LOGUERCIO, A.P., et al. Listeria monocytogenes: um importante patógeno de origem alimentar. Higiene Alimentar, v. 15, n. 80/81, 39-48, 2001.

LOU, Y.; YOUSEF, A.E. Adaptation to sublethal environmental stresses protects Listeria monocytogenes against lethal preservations factors. Applied and Environmental Microbiology. v. 63,n. 4, p.1252-1255, 1997.

LUKINMAA, S., et al. Diversity of Listeria monocytogenes isolates of human and food origin studied by serotyphing automated ribotyping and pulsed-field gel eletrophoresis. Clinical Microbiology and Infection, v.10, 562-568, 2004.

LUNDÉN, J., et al. Adaptive and cross-adaptive responses of persistent and non-persistent Listeria monocytogenes strains to disinfectants. International Journal of Food Microbiology, v. 82, 265-272, 2003.

MANTILLA, S.P.S., et al. Resistência antimicrobiana de bactérias do gênero Listeria spp. Isoladas de carne moída bovina. Brazilian Journal of Veterinary Research and Animal Science, v. 45, n. 2, 116-121, 2008.
MEREGHETTI, L., et al. Low sensitivity of Listeria monocytogenes to quaternary ammonium compounds. Applied and Environmental Microbiology,.v. 66, 5083- 5086, 2000.

MOREIRA, M. R. et al. Inhibitory parameters of essential oils to reduce a foodborne pathogen. LWT - Food Science and Technology. v. 38, p. 565-570, 2005.

NCCLS (National Committee for Clinical Laboratory Standards). Methods for dilution antimicrobial susceptibility tests for bacteria that grow aerobically. Approved standard M7-A6, 2003.

RIBEIRO, A. R., et al. Resistência antimicrobiana em Salmonella enterica subsp enterica sorovar Hadar isoladas de carcaças de frango. Arquivos do Instituto Biológico, v. 73, n 3, 357-360, 2006.

SAMELIS, J., et al. Combinations of nisin with organic acids or salts to control Listeria monocytogeneson sliced pork Bologna stored at $4^{\circ} \mathrm{C}$ in vacuum packages. LWT - Food Science Technology, v.38, 21-28, 2005.

SONI, K.A., et al. The contribution of transcriptomic and proteomic analysis in elucidating stress adaptation responses of Listeria monocytogenes. Foodborne Pathogens and Disease. v. 8, n. 8, p. 843-852, 2011.

SOLOMAKOS, N. et al. The antimicrobial effects of thyme essential oil, nisin, and their combination against Listeria monocytogenes in minced beef during refrigerated storage. Food Microbiology, v. 25, p. 120-127, 2008.

ULTEE, A., et al. Adaptation of the food-borne pathogenBacillus cereus to carvacrol. Archives of Microbiology. v. 174, n.4, p. 233-238, 2000. 